\title{
Resina compuesta en comparación a vidrio ionómero modificado con resina en dientes primarios con caries proximales cavitadas.
}

\section{Composite resin compared to resin modified glass ionomer cement in primary teeth with cavitated proximal caries.}

\begin{abstract}
María José Águila ${ }^{1,2}$, Catalina Toledo ${ }^{1,2}$, Fernanda Alvarado ${ }^{1,2}$, Andrea Cardenas $^{1,2^{*}}$
1. Escuela de Odontología, Facultad de Medicina, Pontificia Universidad Católica de Chile, Santiago, Chile

2. Proyecto Epistemonikos, Santiago, Chile

* Correspondencia Autor: Andrea Cardenas | Dirección: Centro Evidencia UC, Pontificia Universidad Católica de Chile, Diagonal Paraguay 476, Santiago, Chile | E-mail: cardenasandrea77@ gmail.com

RESUMEN

Introducción: Las restauraciones de caries proximales en dientes primarios son complejas de realizar, siendo fundamental la correcta realización de la técnica para la restitución de la dentición funcional. La resina compuesta y vidrio ionómero modificado con resina son de los materiales más utilizados en la actualidad, debido a sus propiedades estéticas y de adhesión, pero aún no está clara la efectividad de un material por sobre otro. Métodos: Para responder la pregunta se realizó una búsqueda en Epistemonikos, la mayor base de datos de revisiones sistemáticas en salud, la cual es mantenida mediante el cribado de múltiples fuentes de información, incluyendo MEDLINE, EMBASE, Cochrane, entre otras. Extrajimos los datos desde las revisiones identificadas, analizamos los datos de los estudios primarios, realizamos un metanálisis y preparamos una tabla de resumen de los resultados utilizando el método GRADE. Resultados y conclusiones: Identificamos nueve revisiones sistemáticas que en conjunto incluyeron siete estudios primarios, de los cuales, cinco corresponden a ensayos aleatorizados. Concluimos que el uso de resina compuesta en caries proximales cavitadas de dientes primarios podría resultar en poca o nula diferencia en el fracaso de la restauración, pero la certeza de la evidencia es baja. Además el uso de resina compuestas probablemente aumente el riesgo de caries secundaria. Además, no se encontraron estudios que evaluaran la retención de la restauración.
\end{abstract}

\section{PALABRAS CLAVE}

Resina compuesta; Vidrio ionómero; Caries proximales; Dientes primarios Epistemonikos; GRADE.

\begin{abstract}
Introduction: Ensure an adequate interproximal caries restorations in primary teeth are essential for the restoration of functional dentition, but the technique is sensitive. Composite resin and resin-modified glass ionomer are among the most widely used ma-terials today, due to their aesthetic and adhesion properties, but the effectiveness of one material over another is not yet clear. Methods: We searched in Epistemonikos, the largest database of systematic reviews in health, which is maintained by screening multiple information sources, including MEDLINE, EMBASE, Cochrane, among others. We extracted data from the systematic reviews, reanalyzed data of primary studies, conducted a meta-analysis and generated a summary of findings table using the GRADE approach. Results and conclusions: We identified nine systematic reviews including seven studies overall, of which five were randomized trials. The use of composite resin may improve the failure of the restorations but the evidence is low. The use of composite resin probably improves the risk of secondary caries. No studies were found evaluating retention of the restoration.
\end{abstract}

\section{KEY WORDS}

Composite resin; Glass ionomer; Proximal caries; Primary teeth; Epistemonikos; GRADE.

Int. J. Inter. Dent Vol. 14(1); 95-99, 2021. 


\section{PROBLEMA}

La caries dental es uno de los problemas orales más prevalentes en el mundo, afectando a más de 530 millones de niños ${ }^{[1]}$. La dentición primaria presenta características que la diferencian con los dientes permanentes en cuanto a su microestructura, grosor y composición del esmalte y la dentina, lo cual puede generar dificultades al restaurar las caries ${ }^{[2]}$. Estas dificultades aumentan cuando se tratan de restauraciones proximales (clase II), ya que al ser de difícil acceso para el operador, el éxito va a depender del grado de cooperación de estos pacientes ${ }^{[3]}$

Es por lo anterior que diferentes materiales han sido utilizados para mejorar los resultados en este tipo de restauraciones y facilitar las técnicas para el operador. Uno de ellos es la resina compuesta, cuyo protocolo de uso incluye grabado ácido selectivo en dentina y esmalte, utilización de un adhesivo y su aplicación de forma incremental. Otro material que ha sido propuesto es el vidrio ionómero modificado con resina, que tiene como ventajas el liberar flúor, ser menos sensible a la humedad y además incluir un menor número de pasos en su aplicación, siendo preferido en aquellos casos donde no se puede aislar con goma dique y/o hay cooperación por parte del paciente $e^{[4]}$.

Este resumen busca evaluar la efectividad de la resina compuesta en las restauraciones proximales en dentición primaria en comparación al vidrio ionómero modificado con resina.

\section{MÉTODOS}

Realizamos una búsqueda en Epistemonikos, la mayor base de datos de revisiones sistemáticas en salud, la cual es mantenida mediante búsquedas en múltiples fuentes de información, incluyendo MEDLINE, EMBASE, Cochrane, entre otras. Extrajimos los datos desde las revisiones identificadas y analizamos los datos de los estudios primarios. Con esta información, generamos un resumen estructurado denominado FRISBEE (Friendly Summaries of Body of Evidence using Epistemonikos), siguiendo un formato preestablecido, que incluye mensajes clave, un resumen del conjunto de evidencia (presentado como matriz de evidencia en Epistemonikos), metanálisis del total de los estudios cuando sea posible, una tabla de resumen de resultados con el método GRADE y una sección de otras consideraciones para la toma de decisión.

\section{Mensajes clave}

- El uso de resina compuesta en caries proximales de dientes primarios podría resultar en poca o nula diferencia en el fracaso de la restauración fracaso de la restauración (baja certeza de la evidencia)

- No se encontraron estudios que evaluaran la retención de la restauración.

- El uso de resina compuesta en caries proximales de dientes primarios, probablemente aumente el riesgo de desarrollar caries secundaria.

\section{Acerca del conjunto de evidencia para esta pregunta}

Encontramos nueve revisiones sistemáticas ${ }^{[4,5,6,7,8,9,10,11,12]}$, que incluyeron seis estudios primarios reportados en siete referencias $[13,14,15,16,17,18,19]$, de los cuales cinco

Cuál es la evidencia

Véase matriz de evidencia en Epistemonikos más abajo.
Qué tipo de pacientes incluyeron los estudios*

Qué tipo de intervenciones incluyeron los estudios*

Qué tipo de desenlaces midieron
Los ensayos ${ }^{[13,14,16,17,18]}$ incluyeron a pacientes entre 3 y 10 años con caries proximales en dentición primaria. Dos ensayos ${ }^{[13,16]}$ incluyeron pacientes con al menos dos caries en dientes primarios, un ensayo ${ }^{[14]}$ a pacientes con al menos tres caries en dientes primarios y otro ensayo ${ }^{[17]}$ a pacientes con al menos una caries en dientes primarios

En todos los ensayos $[13,14,16,17,18]$ los dientes con caries presentaron contacto oclusal con diente antagonista y proximal con diente adyacente.

En tres ensayos ${ }^{[14,16,18]}$ se indicó que los dientes no presentaban signos y síntomas tanto clínica como radiográficamente de patología pulpar o perirradicular.

Un ensayo ${ }^{[14]}$ excluyó a niños no cooperadores, con compromiso médico, deficiente higiene oral, enfermedad gingival o periodontal, mantenedores de espacio y aparatos ortodóncicos.

Todos los ensayos compararon la resina compuesta contra el vidrio ionómero modificado con resina. La utilización de resina en los ensayos implicaba el grabado ácido del tejido dentario, uso de adhesivo y aplicación de resina, mientras que el protocolo para el vidrio ionómero iniciaba con aplicación del primer y posteriormente el material propiamente tal $[13,14,16,17,18]$.

Los ensayos reportaron múltiples desenlaces, los cuales fueron agrupados por las revisiones sistemáticas de la siguiente manera:

- Caries secundaria

- Falla de la restauración

- Adaptación marginal

- Longevidad de la restauración

- Forma anatómica

- Decoloración marginal

- Desgaste del material

El seguimiento varió entre 18 meses $[14,18], 24$ meses $^{[17]}$ y 48 meses ${ }^{[16]}$.

* La información sobre los estudios primarios es extraída desde las revisiones sistemáticas identificadas, no directamente desde los estudios, a menos que se especifique lo contrario.

\section{RESUMEN DE LOS RESULTADOS}

La información sobre los efectos de la resina compuesta en lesiones de caries proximales cavitadas de dientes primarios está basada en cinco ensayos aleatorizados que incluyeron 388 restauraciones (215 pacientes)

Cuatro ensayos midieron el desenlace caries secundaria [13,14,16,17], y tres ensayos midieron falla de la restauración $[16,17,18]$. El desenlace retención no fue reportado con datos cuantitativos ni narrativos.

El resumen de los resultados es el siguiente:

- El uso de resina compuesta en caries proximales de dientes primarios podría podría resultar en poca o nula diferencia en el fracaso de la restauración (certeza de la evidencia baja).

- No se encontraron estudios que evaluaran la retención de la restauración.

- El uso de resina compuesta en caries proximales de dientes primarios, probablemente aumente la formación de caries secundaria. 


\section{Resina compuesta comparada con vidrio ionómero modificado con resina en rlesiones priximales en dentición primaria}

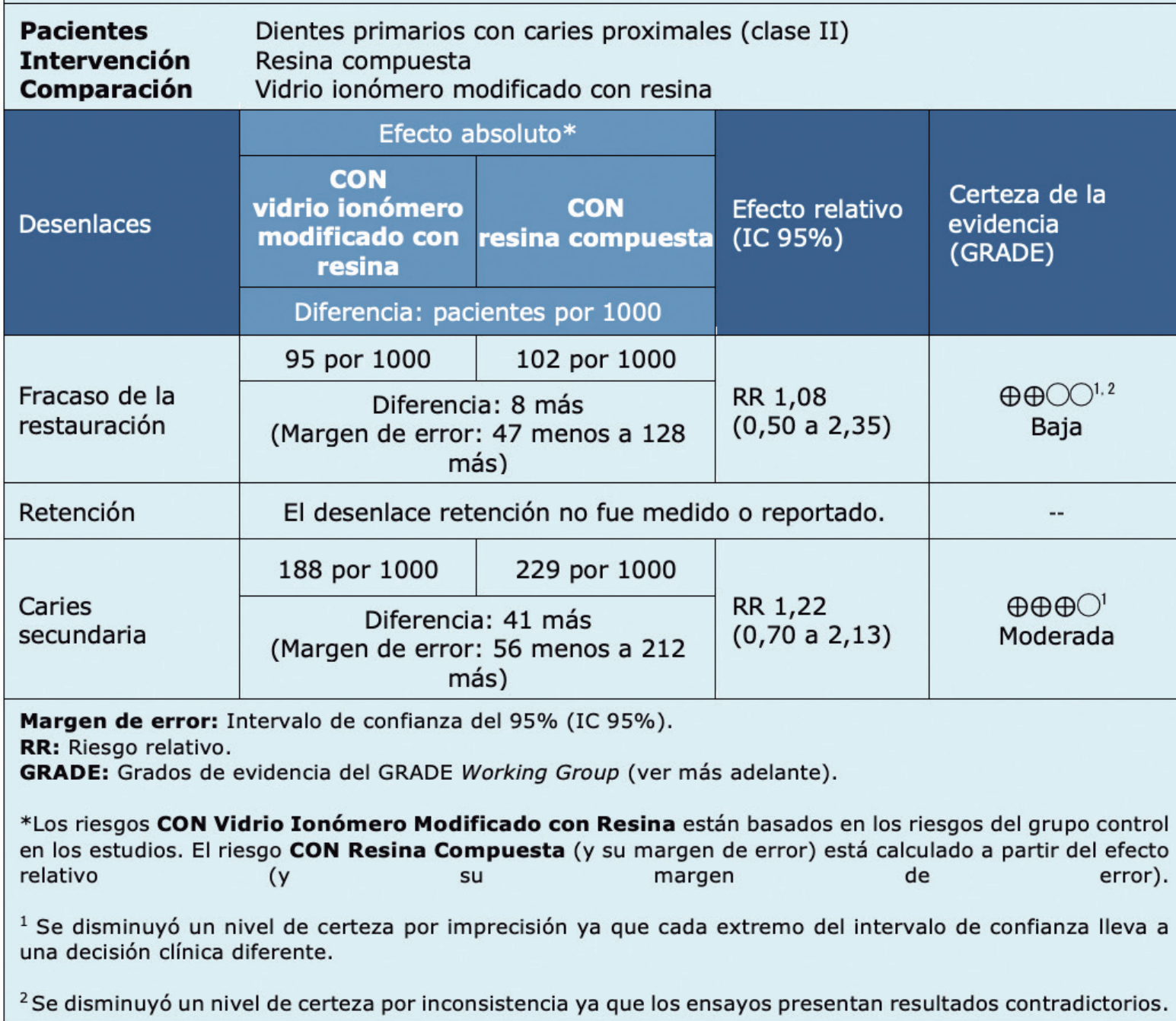

Siga el enlace para acceder a la versión interactiva de esta tabla (Interactive Summary of Findings - iSoF)

\begin{tabular}{|l|}
\hline Acerca de la certeza de la evidencia (GRADE)* \\
\hline$\bigoplus \bigoplus \bigoplus$ \\
Alta: La investigación entrega una muy buena indicación del efecto probable. La probabilidad de que \\
el efecto sea sustancialmente distinto† es baja. \\
$\bigoplus \bigoplus \bigoplus \bigcirc$ \\
Moderada: La investigación entrega una buena indicación del efecto probable. La probabilidad de \\
que el efecto sea sustancialmente distinto’ es moderada. \\
$\bigoplus \bigoplus \bigcirc \bigcirc$ \\
Baja: La investigación entrega alguna indicación del efecto probable. Sin embargo, la probabilidad \\
de que el efecto sea sustancialmente distinto† es alta. \\
$\bigoplus \bigcirc \bigcirc \bigcirc$ \\
Muy baja: La investigación no entrega una estimación confiable del efecto probable. La probabilidad \\
de que el efecto sea sustancialmente distinto† es muy alta. \\
\hline *Esto es también denominado 'calidad de la evidencia' o 'confianza en los estimadores del efecto'. \\
tSustancialmente distinto = una diferencia suficientemente grande como para afectar la decisión \\
\hline
\end{tabular}




\section{OTRAS CONSIDERACIONES PARA LA TOMA DE DECISIÓN}

\section{A quién se aplica y a quién no se aplica esta evidencia}

- Los resultados de este resumen son aplicables en pacientes con caries proximales en dientes primarios.

- Esta evidencia no aplicaría en aquellos pacientes con caries proximal en dentición primaria que tengan una destrucción coronaria severa, movilidad dentaria o piezas dentarias próximas a exfoliar.

\section{Sobre los desenlaces incluidos en este resumen}

- Los desenlaces seleccionados son considerados críticos para la toma de decisión de acuerdo a la opinión de los autores de este resumen, coincidiendo en general con los evaluados en las revisiones sistemáticas identificadas.

- El desenlace caries secundaria es crítico para la toma de decisiones ya que determina el fracaso de la restauración, necesidad de recambio y por lo tanto un nuevo tratamiento.

- El desenlace retención entendido como la pérdida de la restauración, es crítico para la toma de decisiones por la importancia que tiene para los pacientes. Sin embargo, este desenlace no fue reportado por las revisiones sistemáticas.

\section{Balance daño/beneficio y certeza de la evidencia}

- La evidencia muestra poca o nula diferencia en el fracaso de la restauración al comparar resina compuesta contra vidrio ionómero modificado con resina, pero la certeza de la evidencia es baja.

- Por otro lado, es probable que el uso de resina compuesta aumente el riesgo de desarrollar caries secundarias comparado con el uso de vidrio ionómero modificado con resina.

- Pese a que el balance podría estar a favor del uso de vidrio ionómero modificado con resina, la decisión de elegir un material sobre otro debe individualizarse según el tipo de paciente, su edad, el grado de cooperación, riesgo cariogénico, las condiciones de trabajo, las posibilidades de realizar aislación absoluta, entre otros, considerando que la resina compuesta requiere de un buen control de la humedad, siendo una técnica sensible.

\section{Consideraciones de recursos}

- Ninguno de los estudios incluidos realizó un análisis de costos entre el uso de resina compuesta y vidrio ionómero modificado con resina en restauraciones proximales en dentición primaria.

- El protocolo para restauraciones de resina compuesta incluye el uso de aislación con goma dique, la aplicación de ácido, adhesivo y la resina, por lo que sería más costoso que el vidrio ionómero modificado con resina.

\section{Qué piensan los pacientes y sus tratantes}

- Enfrentados con la evidencia disponible, la mayoría de los pacientes y tratantes deberían inclinarse a favor del uso de vidrio ionómero para las restauraciones proximales en dientes permanentes.

- El vidrio ionómero modificado con resina suele ser preferido en pacientes poco cooperadores o donde no es posible aislar con goma dique. Sumado, a su menor tiempo clínico se considera favorable en el tratamiento de pacientes pediátricos.

- El uso de resina compuesta, por su parte, implica mayor cantidad de materiales y pasos para su realización junto con una mayor sensibilidad de la técnica, por lo que su uso dependerá de la experiencia y preferencias del tratante junto a la disponibilidad de recursos del centro de atención y/o del paciente. En situaciones donde existe un correcto control de la humedad la resina compuesta debiese ser el material de elección.

\section{Diferencias entre este resumen y otras fuentes}

- Las conclusiones de este resumen concuerdan con ocho de las revisiones sistemáticas identificadas $[4,6,7,8,9,10,11,12]$, las cuales consideran que la resina compuesta y el vidrio ionómero modificado con resina tienen un desempeño clínico similar, excepto en relación a la formación de caries secundaria, donde el vidrio ionómero modificado con resina tiene mejores resultados

- Solo una revisión ${ }^{[5]}$ concluyó que el desempeño de la resina compuesta es mejor al del vidrio ionómero, sin embargo esta revisión no incluyó el total de estudios incluidos en este resumen de evidencia.

- La American Academy of Pediatric Dentistry ${ }^{[20]}$ señala que está recomendado el uso de resina compuesta en restauraciones proximales en dientes primarios, sin embargo recomienda no utilizar este materia en aquellos casos donde no se pueda aislar o se trate de un paciente no cooperador. En relación al vidrio ionómero modificado con resina señala que la opinión de expertos apoya su uso en restauraciones proximales en dientes primarios.

\section{¿Puede que cambie esta información en el futuro?}

- La probabilidad de que futura investigación cambie las conclusiones de este resumen es alta debido a la incertidumbre presente en la mayoría de los desenlaces evaluados. Sólo en el caso del desenlace caries secundaria se considera que las conclusiones podrían cambiar, puesto que la certeza de la evidencia es moderada.

- No se identificó ningún ensayo aleatorizado en curso en International Clinical Trials Registry Platform de la Organización Mundial de la Salud $n$ revisiones sistemáticas en International prospective register of systematic reviews (PROSPERO) del National Institute for Health Research.

\section{CÓMO REALIZAMOS ESTE RESUMEN}

Mediante métodos automatizados y colaborativos recopilamos toda la evidencia relevante para la pregunta de interés y la presentamos en una matriz de evidencia.

Siga el enlace para acceder a la versión interactiva: Resina compuesta en comparación a vidrio ionómero modificado con resina en restauraciones proximales en dentición primaria.

\section{NOTAS}

Si con posterioridad a la publicación de este resumen se publican nuevas revisiones sistemáticas sobre este tema, en la parte superior de la matriz se mostrará un aviso de "nueva evidencia".

Este artículo es parte del proyecto síntesis de evidencia de Epistemonikos. Se elabora con una metodología preestablecida, siguiendo rigurosos estándares metodológicos y proceso de revisión por pares interno. Cada uno de estos artículos corresponde a un resumen, denominado FRISBEE (Friendly Summary of Body of Evidence using Epistemonikos), cuyo principal objetivo es sintetizar el conjunto de evidencia de una pregunta específica, en un formato amigable a los profesionales clínicos. Sus principales recursos se basan en la matriz de evidencia de Epistemonikos y análisis de resultados usando metodología GRADE. Mayores detalles de los métodos para elaborar este FRISBEE están descritos aquí (http://dx.doi.org/10.5867/medwave.2014.06.5997)

La Fundación Epistemonikos es una organización que busca acercar la información a quienes toman decisiones en salud, mediante el uso de tecnologías. Su principal desarrollo es la base de datos Epistemonikos (www.epistemonikos.org).

\section{DECLARACIÓN DE CONFLICTOS DE INTERESES}

Los autores declaran no tener conflictos de intereses con la materia de este artículo.

\section{AGRADECIMIENTOS}

Este resumen de evidencia fue elaborado con el apoyo metodológico del Centro Evidencia UC, Facultad de Medicina, Pontificia Universidad Católica de Chile. 


\section{Bibliografía}

1 Organización Mundial de la Salud. Salud bucodental. [Internet]. 2020. https://www. who.int/es/news-room/fact-sheets/detail/oral-health

2. Monsalves S, Terrazas P, Toro G, Bader M. Evaluación del grado de sellado marginal y resistencia adhesiva de restauraciones de resina compuesta con adhesivo convencional en dentición primaria y definitiva. Rev Clin Periodoncia Implantol Rehabil Oral. [Internet]. 2014. 7(3): 149-56. https://doi.org/10.1016/j. piro.2014.09.001

3. Marró M, Cabello R, Rodríguez G, Mustakis A, Urzúa I. Tratamiento de lesiones de caries interproximales mediante el uso de infiltrantes. Rev Clin Periodoncia Implantol Rehabil Oral. [Internet]. 2011;4(3):134-7. Disponible en: https://scielo. conicyt.cl/scielo.php?script=sci_arttext\&pid=S0719-01072011000300011\&lng=es. 4. Dias AGA, Magno MB, Delbem ACB, Cunha RF, Maia LC, Pessan JP. Clinica performance of glass ionomer cement and composite resin in Class II restorations in primary teeth: A systematic review and meta-analysis. J Dent. [Internet]. 2018 [cited 2020 Oct 30];73:1-13. Available from: https://pubmed.ncbi.nlm.nih.gov/29649506/ 5. Souza ALM de, Magalhães TC, Lopes AG, Carlo HL, Santos RL dos, Carlo FG de C. Qual material possui maior longevidade em molares decíduos: resina composta ou cimento de ionômero de vidro? - revisão sistemática da literatura HU rev [Internet]. 2018 [cited 2020 Oct 30]: 115-22. Available from: http://ojs2.ufjf. emnuvens.com.br/hurevista/article/view/13975/pdf

6. Ortiz-Ruiz AJ, Pérez-Guzmán N, Rubio-Aparicio M, Sánchez-Meca J. Success rate of proximal tooth-coloured direct restorations in primary teeth at 24 months: a meta-analysis. Sci Rep. [Internet]. 2020 Apr 14;10(1): 6409. Available from: https:// pubmed.ncbi.nlm.nih.gov/32286461

7. Raggio DP, Tedesco TK, Calvo AFB, Braga MM. Do glass ionomer cements prevent caries lesions in margins of restorations in primary teeth? A systematic review and meta-analysis. J Am Dent Assoc. [Internet]. 2016 [cited 2020 Oct 30];147:177-85. Available from: http://jada.ada.org/article/S0002817715009873/fulltext

8. Chisini LA, Collares K, Cademartori MG, de Oliveira LJC, Conde MCM, Demarco $\mathrm{FF}$, et al. Restorations in primary teeth: a systematic review on survival and reasons for failures. Int J Paediatr Dent. [Internet]. 2018 [cited 2020 Oct 30]; 28:123-39. Available from: https://pubmed.ncbi.nlm.nih.gov/29322626/

9. Ruengrungsom C, Palamara JEA, Burrow MF. Comparison of ART and conventional techniques on clinical performance of glass-ionomer cement restorations in load bearing areas of permanent and primary dentitions: A systematic review. J Dent. 2018;78:1-21.

10. Dias AGA. Cimento de ionômero de vidro é melhor do que resina composta em restaurações classe II de dentes decíduos? Uma revisão sistemática com meta- análise. 2016 [cited 2020 Oct 30];71-1. Available from: https://repositorio.unesp.br/ handle/11449/148761

11. Pires CW, Pedrotti D, Lenzi TL, Soares FZM, Ziegelmann PK, Rocha R de O. Is there a best conventional material for restoring posterior primary teeth? A network meta-analysis. Braz Oral Res [Internet]. 2018 Mar 1 [cited 2020 Oct 30];32:e10. Available from: https://pubmed.ncbi.nlm.nih.gov/29513886/

12. Santos AP, Moreira IK, Scarpelli AC, Pordeus IA, Paiva SM, Martins CC. Survival of Adhesive Restorations for Primary Molars: A Systematic Review and Metaanalysis of Clinical Trials. Pediatr Dent. 2016 Oct 15;38(5):370-8. PMID: 28206891.

13 dos Santos MP Passos M, Luiz RR, Maia LC. A randomized trial of resin-based restorations in class I and class II beveled preparations in primary molars: 24-month results. J Am Dent Assoc. 2009 Feb;140(2):156-66; quiz 247-8. doi: 10.14219/jada. archive.2009.0129. PMID: 19188412.

14. Bektas Donmez S, Uysal S, Dolgun A, Turgut MD. Clinical performance of aesthetic restorative materials in primary teeth according to the FDI criteria. Eur J Paediatr Dent. 2016 Sep;17(3):202-12. PMID: 27759409.

15. Pinto Gdos S, Oliveira LJ, Romano AR, Schardosim LR, Bonow ML, Pacce M, Correa MB, Demarco FF, Torriani DD. Longevity of posterior restorations in primary teeth: results from a paediatric dental clinic. J Dent. 2014 Oct; 42(10): 1248-54. doi: 10.1016/j.jdent.2014.08.005. Epub 2014 Aug 20. PMID: 25150105.

16. Alves dos Santos MP, Luiz RR, Maia LC. Randomised trial of resin-based restorations in Class I and Class II beveled preparations in primary molars: 48-month results. J Dent. 2010 Jun;38(6):451-9. doi: 10.1016/j.jdent.2010.02.004. Epub 2010 Feb 25. PMID: 20188783.

17. Fuks AB, Araujo FB, Osorio LB, Hadani PE, Pinto AS. Clinical and radiographic assessment of Class II esthetic restorations in primary molars. Pediatr Dent. 2000 Nov-Dec;22(6):479-85. PMID: 11132507.

18. Casagrande L, Dalpian DM, Ardenghi TM, Zanatta FB, Balbinot CE, GarcíaGodoy F, De Araujo FB. Randomized clinical trial of adhesive restorations in primary molars. 18-month results. Am J Dent. 2013 Dec;26(6):351-5. PMID: 24640441.

19. Sengul F, Gurbuz T. Clinical Evaluation of Restorative Materials in Primary Teeth Class II Lesions. J Clin Pediatr Dent. 2015 Summer;39(4):315-21. doi: 10.17796/1053-4628-39.4.315. PMID: 26161601.

20. American Academy of Pediatric Dentistry. Pediatric restorative dentistry. The Reference Manual of Pediatric Dentistry. Chicago. American Academy of Pediatric Dentistry; 2019: 341-53. Available from: https://www.aapd.org/globalassets/media/ policies_guidelines/bp_restorativedent.pdf 